\title{
Impacto del sobrepeso y la obesidad en el asma infantil
}

\author{
ALBERTO VIDAL G.*, ANA MARÍA ESCOBAR C.*, ELIANA CERUTI D.*, \\ MARÍA TERESITA HENRÍQUEZ Y.* y MARÍA EUGENIA MEDINA R.***
}

\section{Impact of the overweight and obesity in the pediatric asthma}

Introduction: Evidence suggests that overweight and obesity alter the control and lung function in asthmatic children. Aim: Measure the impact of overweight and obesity in the control and lung function of a population of persistent asthma in children. Material and Method: Nutritional evaluation, asthma control and spirometry were performed in patients with persistent asthma, 5 to 15 years of age controlled in a pediatric respiratory unit. We determined the presence of asthma symptoms, specific parameters for the management and predisposing factors according to the nutritional status. Differences between groups were evaluated using chi-square Mantel-Haenszel and trend; a $p<0.05$ was considered significant. Results: 219 patientes were studied, males 60.3\%, average age of 8.5 yearsold. The frequency of overweight and obesity reached $63.5 \%$. Normal weight $36.5 \%$, overweight $30.6 \%$ and obese $32.9 \%$. Uncontrolled asthma (normal weight $37.5 \%$, with overweight and obese $65.5 \%, p$ $=0.0001$ ), abnormal spirometry (normal weight $27.5 \%$, with overweight and obese $45.3 \%, p=0.01$ ). Uncontrolled asthma linear trend (normal weight $37.5 \%$, overweight $67.2 \%$, obese $63.9 \%, p=0.0008$ ). Abnormal spirometry linear trend $(27.5 \%, 46.3 \%, 44.4 \%, p=0.03)$. Conclusions: The asthmatics with overweight and obesity have a bigger proportion of uncontrolled asthma and abnormal spirometry than the asthmatics with normal weight. It exists a trend of uncontrolled asthma and abnormal spirometry when passing from one category to another of the nutritional classification.

Key words: Asthma control, lung function, overweight, obese.

\section{Resumen}

Introducción: La evidencia sugiere que el sobrepeso y la obesidad alterarían el control y la función pulmonar de los niños asmáticos. Objetivo: Medir el impacto del sobrepeso y obesidad en el control y función pulmonar de una población de niños asmáticos persistentes. Material y Método: Se realizó evaluación nutricional, medición de control de asma y espirometría a niños asmáticos persistentes entre 5 y 15 años, controlados en una Unidad de Enfermedades Respiratorias. Se determinó la presencia de síntomas de asma, los parámetros que permiten precisar su adecuado manejo y algunos factores predisponentes según estado nutricional. Las diferencias entre grupos se evaluaron a través del chi cuadrado de Mantel-Haenszel y el de tendencia, considerándose significativos valores de $p<0,05$. Resultados: Se estudiaron a 219 niños, sexo masculino 60,3\%, promedio de edad de 8,5 años. La frecuencia de sobrepeso y obesidad alcanzó el 63,5\%. Asmáticos eutróficos 36,5\%, con sobrepeso 30,6\% y obesos 32,9\%. Asma no controlada (eutróficos 37,5\%, con sobrepeso y obesidad 65,5\%, p =0,0001), espirometría alterada (eutróficos $27,5 \%$, sobrepeso y obesidad 45,3\%, $p=0,01$ ). Tendencia lineal en asma no controlada (eutróficos $37,5 \%$, sobrepeso $67,2 \%$, obesos $63,9 \% p=0.0008$ ). Tendencia lineal en espirometría alterada (27,5\%, 46,3\%, 44,4\%, $p=0,03)$, Conclusiones: Los asmáticos con sobrepeso y obesidad tienen una proporción significativamente mayor de asma no controlada y de espirometría alterada, que los asmáticos con estado nutricional normal. Existe una tendencia al aumento en las proporciones de asma no controlada y espirometría alterada al pasar de una categoría a otra de la clasificación nutricional.

Palabras clave: Control de asma, función pulmonar, sobrepeso, obesidad.

* Pediatra Especialista en Enfermedades Respiratorias. Hospital de niños Roberto del Río, Santiago de Chile.

** Tecnólogo Médico, Unidad de Enfermedades Respiratorias, Hospital de niños Roberto del Río, Santiago de Chile. 


\section{Introducción}

En las últimas cuatro décadas el asma bronquial, el sobrepeso y la obesidad han experimentado un aumento sostenido de sus prevalencias. Datos obtenidos en Estados Unidos por la National Health and Nutrition Examination Survey (NHANES), demuestran que entre los años 1976 y 2006, la obesidad aumentó de 6,5\% a $17 \%$ en niños de 6 a 11 años; mientras que el grupo de adolescentes de 12 a 19 años, subió de $5 \%$ a $17,6 \%$. Con el sobrepeso ha ocurrido algo similar, alcanzando $33,3 \%$ en niños de 6 a 11 años y $34,1 \%$ en adolescentes de 12 a 19 años ${ }^{1-}$ ${ }^{3}$. Un estudio chileno publicado en el año 2004, notificó el incremento de sobrepeso y obesidad en ambos sexos, especialmente para las mujeres, con similar magnitud en la población escolar y adolescente. La obesidad en mujeres prepuberales $(6$ a 9 años) alcanzó el 24\% y el sobrepeso 16,2\%. En las mujeres adolescentes (10 a 16 años) se encontró $17,6 \%$ de obesidad y $24,9 \%$ de sobrepeso. En los hombres prepuberales (6 a 11 años) se encontró obesidad en $29,5 \% \%$ y sobrepeso en $19,6 \%$. Los varones adolescentes (12 a 16 años) tenían $14,6 \%$ de obesidad y $14,6 \%$ de sobrepeso ${ }^{4}$.

En asma, los estudios mundiales ISAAC (Fase I a III) confirman un incremento en el diagnóstico de sibilancias en los últimos 12 meses. Cabe destacar que este aumento ha sido menor en los países de habla inglesa y del Noroeste Europeo y mayor en África, parte de Asia y Latinoamérica, zonas consideradas inicialmente de baja prevalencia, lo que se explica por un mejor diagnóstico de la enfermedad en los últimos años. La prevalencia de asma pediátrica en Latinoamérica aumenta cada año a razón de $0,07 \%$ en niños de 6 a 7 años y $0,32 \%$ en adolescentes de 13 a 14 años ${ }^{5}$. La versión chilena del estudio ISAAC (1994-2002), confirma el incremento de la prevalencia de asma, así como de los episodios severos de asma en los adolescentes de 13 a 14 años ${ }^{6}$.

Varios aspectos de la relación entre asma y obesidad han concertado el interés de investigaciones recientes, la mayoría con resultados aún controversiales. Entre ellos destacan la mayor incidencia de asma en niñas obesas prepuberales, la relación temporal del sobrepeso y la obesidad como predictores y/o antecesores de asma, genes comunes entre obesidad y asma, el efecto proinflamatorio de la obesidad mediante las adipoquinas, la influencia de hormonas implicadas en el metabolismo energético como Leptina, el efecto de la programación fetal, nutrición prenatal y actividad física en el desarrollo posterior de obesidad y asma ${ }^{7-14}$.
Estudios realizados en adultos demostraron que los pacientes con asma y sobrepeso u obesidad, tienen peor control de la enfermedad, peor calidad de vida y menor respuesta al tratamiento farmacológico, en especial cuando el índice de masa corporal (IMC) supera los $25 \mathrm{~kg} / \mathrm{m}^{2} 15-18$. En niños, la evidencia no es tan concluyente, sin embargo, publicaciones recientes reportan mayor prevalencia de obesidad en las consultas de urgencia por crisis de asma al compararlas con consultas por otras causas y peor calidad de vida en niños asmáticos con mayor peso a la edad escolar que aquellos con peso normal e incluso que los niños con sobrepeso sin asma ${ }^{19,20}$. Por el creciente interés a nivel mundial y la escasa literatura nacional, nos propusimos desarrollar una investigación centrada en medir el impacto que produce el sobrepeso y la obesidad en el control de síntomas y la función pulmonar de un grupo de niños asmáticos persistentes en control por la especialidad.

\section{Material y Método}

Estudio descriptivo transversal realizado en la Unidad de Enfermedades Respiratorias del Hospital Roberto del Río. Se enrolaron a niños y adolescentes entre 5 y 15 años con diagnóstico de asma persistente leve, moderada o severa. El período del estudio fue de marzo de 2009 hasta abril de 2010. Todos los pacientes fueron sometidos a una espirometría, evaluación nutricional y medición de control de síntomas del asma. Se excluyeron a los pacientes con asma y otra comorbilidad respiratoria crónica, también a los pacientes con bajo peso (IMC $<10)$ y a aquellos con contraindicaciones para realizar el estudio respiratorio funcional.

El tamaño muestral fue calculado con un poder de $80 \%$ y un error aceptable de $5 \%$. Se realizó una comparación de la frecuencia de síntomas de asma y de alteraciones de la función pulmonar según el estado nutricional. Las diferencias fueron establecidas con los test chi cuadrado de MantelHaenszel con corrección de Yates y de tendencia lineal, considerando diferencia significativa un valor de $\mathrm{p}<0,05$.

En la evaluación nutricional se utilizó la norma nacional vigente, que utiliza el IMC, con percentil (p) de acuerdo a edad y estadío de desarrollo puberal de Tanner ${ }^{21}$. Se utilizó una balanza con escalímetro marca Health o Meter, modelo $402 \mathrm{KL}$, que fue calibrada diariamente antes de las mediciones. Luego de la evaluación se dividió la muestra en tres categorías nutricionales: asmáticos eutróficos (IMC p10 a 84), asmáticos 
con sobrepeso (IMC p85-94) y asmáticos con obesidad (IMC $\mathrm{p} \geq 95$ ).

El control del asma se midió por entrevista al cuidador y al niño o adolescente, utilizando el cuestionario de control de síntomas de asma recomendado por la Iniciativa global para el asma (GINA) en su versión 2010, para niños mayores de 5 años. Este cuestionario mide la frecuencia de síntomas diurnos o nocturnos, del uso de la medicación de rescate, de la limitación de las actividades cotidianas, de las exacerbaciones e incorpora además la evaluación de la función pulmonar mediante el registro del volumen espiratorio forzado en el primer segundo o $\mathrm{VEF}_{1}{ }^{22}$. La espirometría fue realizada por un médico especializado en estudio respiratorio funcional, con un espirómetro Jaeger modelo MS-PFT. Para la realización e interpretación del estudio funcional se siguieron las recomendaciones de consensos nacionales ${ }^{23,24}$. Sólo para fines de la investigación, se utilizó el término "espirometría alterada", para definir aquellas cuyo informe mostró alteración obstructiva en cualquiera de sus grados (mínima, leve, moderada o severa). El resto del análisis de la espirometría se realizó con los parámetros habitualmente utilizados en el control del asma (\% $\mathrm{VEF}_{1}$, relación $\mathrm{VEF}_{1} / \mathrm{CVF}, \mathrm{FEF}_{25-75 \%}$, aumento significativo post-salbutamol de $\mathrm{VEF}_{1}$ y $\mathrm{FEF}_{25-75 \%}$ ). Se realizó un cuestionario adicional a los padres y adolescentes para detectar otras condiciones relacionadas con mal control de asma en el último mes y se revisó la técnica inhalatoria en la entrevista. Se consideró mala adherencia el uso menor del $80 \%$ de las dosis de tratamiento controlador indicado en un mes. Las otras condiciones evaluadas fueron: síntomas riníticos, síntomas de reflujo gastroesofágico, tabaquismo pasivo, polvo de habitación, industrias contaminantes cercanas, humedad interior u hongos, calefacción contaminante, mascotas en el hogar, condición de estrés en niño percibida por el cuidador y uso de fármacos de conocida contraindicación en asma bronquial. Se revisaron los registros clínicos para obtener la severidad de asma persistente según GINA, clasificación utilizada por consenso en nuestro grupo. Se registró además el tipo de tratamiento controlador utilizado y el uso de dosis elevadas de corticoide inhalatorio para la edad definido según GINA versión 2010 como el uso diario de budesonida $>400 \mu \mathrm{g}$ o fluticasona $>500 \mu \mathrm{g}$ en niños de 5 o más años ${ }^{22}$.

\section{Resultados}

Se enrolaron a 223 pacientes asmáticos persistentes. Cuatro pacientes $(1,7 \%)$ fueron excluidos del estudio: dos correspondieron a niños de 5 años que por su edad no lograron entender la maniobra para la espirometría y los otros dos tenían IMC $<$ al p10, en los que además se sospechó fibrosis quística, siendo referidos a sus médicos tratantes para completar estudio. Las pérdidas no influyeron en el análisis estadístico final por lo que no fueron consideradas.

De los 219 pacientes que finalmente fueron analizados, el $60,3 \%$ correspondió al género masculino, con un promedio de 8,5 años (101,9 meses). Según la clasificación de severidad de asma de GINA encontramos: $19,2 \%$ persistentes leves, $59,8 \%$ persistentes moderados y $21 \%$ persistentes severos.

El 55,3\% de la serie no logró el control de la enfermedad según la encuesta de control GINA. El 38,8\% (85 pacientes) presentó espirometría con algún grado de alteración. La alteración predominante fue obstructiva: limitación obstructiva mínima 91,8\%, limitación obstructiva leve $8 \%$ y limitación obstructiva moderada $1,2 \%$. No se encontraron pacientes con limitación obstructiva severa. Sólo dos pacientes tenían capacidad vital forzada $(\mathrm{CVF})<80 \%$, ambos en rangos de limitación restrictiva leve asociada a limitación obstructiva moderada, lo que explica esta disminución. El 53\% de los pacientes se trataba sólo con corticoides inhalados, el $47 \%$ con mezcla de corticoide y beta 2 agonista de acción prolongada. El 6,8\% de los pacientes recibía dosis elevadas de corticoide inhalado.

La frecuencia de sobrepeso y obesidad alcanzó el 63,5\%. Las distribución según el estado nutricional fue: $36,5 \%$ eutróficos, $30,6 \%$ sobrepeso y $32,9 \%$ obesos. El porcentaje global de sobrepeso y obesidad fue significativamente mayor en el grupo de varones asmáticos que en las mujeres asmáticas $(68,9 \%$ vs $55,2 \%$ respectivamente, $\mathrm{p}=0,03)$. Al distribuir la serie en tres rangos etarios, se mantuvo la tendencia de mayor proporción de sobrepeso y obesidad en los varones. Sobrepeso y obesidad de 5 a 7 años $(66,2 \%$ hombres, 33,8\% mujeres), de 8 a 11 años (63,5\% en hombres, $33,5 \%$ mujeres) y de 12 a 15 años (68,4\% en hombres, $31,6 \%$ en mujeres).

La Tabla 1 muestra las características generales del grupo de asmáticos distribuidos según el estado nutricional, destacando las condiciones relacionadas con inadecuado control de asma. En ambas categorías nutricionales se aprecian altas frecuencias de algunos factores modificables asociados al mal control como la mala adherencia al tratamiento, mala técnica inhalatoria, los síntomas riníticos y la presencia de mascotas en el hogar. 
A excepción del mayor porcentaje de varones y de una mejor adherencia al tratamiento inhalatorio de mantención en los asmáticos con exceso de peso (sobrepeso u obesidad), no se encontraron diferencias importantes entre ambos grupos.
La Tabla 2 muestra las diferencias en el control de síntomas, función pulmonar y tratamiento de los niños según dos categorías nutricionales: asmáticos eutróficos y asmáticos con exceso de peso. Se demuestra que los pacientes asmáticos

Tabla 1. Características generales en 219 niños y adolescentes asmáticos persistentes según su estado nutricional

\begin{tabular}{|c|c|c|c|c|c|}
\hline & \multicolumn{2}{|c|}{$\begin{array}{l}\text { Amáticos eutróficos } \\
\qquad(\mathrm{n}=\mathbf{8 0})\end{array}$} & \multicolumn{2}{|c|}{$\begin{array}{l}\text { Asmáticos con exceso de peso } \\
\qquad(\mathrm{n}=139)\end{array}$} & \multirow[t]{2}{*}{$\mathbf{p}$} \\
\hline & $\mathbf{n}$ & $(\%)$ & $\mathbf{n}$ & $(\%)$ & \\
\hline Hombres & 41 & $(51,3)$ & 91 & $(65,5)$ & 0,03 \\
\hline Asma severa & 17 & $(21,2)$ & 29 & $(20,9)$ & 0,94 \\
\hline Técnica inhalatoria deficiente & 25 & $(31,3)$ & 44 & $(31,7)$ & 0,95 \\
\hline Mala adherencia & 40 & $(50)$ & 49 & $(35,3)$ & 0,03 \\
\hline Síntomas de rinitis alérgica & 66 & $(82,5)$ & 114 & $(82)$ & 0,93 \\
\hline Tabaquismo pasivo & 44 & $(55)$ & 66 & $(47,5)$ & 0,28 \\
\hline Polvo de habitación & 19 & $(23,8)$ & 26 & $(18,7)$ & 0,37 \\
\hline Humedad & 13 & $(16,1)$ & 31 & $(22,3)$ & 0,28 \\
\hline Calefacción contaminante & 16 & (20) & 22 & $(15,8)$ & 0,61 \\
\hline Industrias contaminantes & 14 & $(17,5)$ & 25 & $(17,9)$ & 0,92 \\
\hline Mascotas & 55 & $(68,8)$ & 85 & $(61,2)$ & 0,26 \\
\hline Estrés & 17 & $(21,3)$ & 36 & $(25,9)$ & 0,44 \\
\hline Síntomas de reflujo gastroesofágico & 8 & $(10)$ & 10 & $(7,2)$ & 0,47 \\
\hline Fármacos contraindicados & 2 & $(2,5)$ & 2 & $(1,4)$ & 0,62 \\
\hline
\end{tabular}

Tabla 2. Aspectos relacionados con el control del asma y función pulmonar en 219 niños y adolescentes según su estado nutricional.

\begin{tabular}{|c|c|c|c|c|c|}
\hline & \multicolumn{2}{|c|}{$\begin{array}{l}\text { Amáticos eutróficos } \\
\qquad(\mathrm{n}=\mathbf{8 0})\end{array}$} & \multicolumn{2}{|c|}{$\begin{array}{l}\text { Asmáticos con } \\
\text { exceso de peso } \\
(n=139)\end{array}$} & \multirow[t]{2}{*}{$\mathbf{p}$} \\
\hline & n & $(\%)$ & n & $(\%)$ & \\
\hline Asma no controlada (GINA) & 30 & $(37,5)$ & 91 & $(65,5)$ & 0,0001 \\
\hline Síntomas diurnos ( $\geq 2$ veces a la semana) & 45 & $(56,3)$ & 99 & $(71,1)$ & 0,03 \\
\hline Síntomas nocturnos & 55 & $(68,8)$ & 105 & $(75,5)$ & 0,35 \\
\hline Tos con ejercicio & 62 & $(77,5)$ & 119 & $(85,6)$ & 0,18 \\
\hline Consultas en urgencia por crisis de asma & 26 & $(32,5)$ & 50 & $(36)$ & 0,7 \\
\hline Hospitalizaciones por crisis de asma & 10 & $(12,5)$ & 16 & $(11,5)$ & 0,82 \\
\hline Dosis elevadas de corticoide inhalatorio (GINA) & 5 & $(6,3)$ & 10 & $(7,2)$ & 0,99 \\
\hline Uso de corticoides más $\beta 2$ agonista de acción prolongada & 36 & $(45)$ & 67 & $(48,2)$ & 0,75 \\
\hline Espirometría alterada & 22 & $(27,5)$ & 63 & $(45,3)$ & 0,01 \\
\hline $\mathrm{VEF}_{1}<80 \%$ & 4 & $(5)$ & 4 & $(2,9)$ & 0,66 \\
\hline Relación $\mathrm{VEF}_{1} / \mathrm{CVF}<80 \%$ & 12 & $(15)$ & 44 & $(31,7)$ & 0,01 \\
\hline $\mathrm{FEF}_{25-75}<\mathrm{LIN}$ & 23 & $(28,8)$ & 63 & $(45,3)$ & 0,02 \\
\hline Cambio $\mathrm{VEF}_{1} \geq 12 \%$ post-salbutamol & 14 & $(17,5)$ & 37 & $(26,6)$ & 0,17 \\
\hline Cambio $\geq 30 \% \mathrm{FEF}_{25-75}$ post-salbutamol & 23 & $(28,8)$ & 43 & $(30,9)$ & 0,85 \\
\hline
\end{tabular}

$\mathrm{VEF}_{1}$ : volumen espiratorio forzado en el primer segundo, CVF: capacidad vital forzada, $\mathrm{FEF}_{25-75}$ : flujo espiratorio forzado al $25-75 \%$ de la capacidad vital forzada, LIN: límite inferior de normalidad. 
Tabla 3. Aspectos relacionados con el control del asma y función pulmonar en 219 niños y adolescentes según la severidad de la patología nutricional

\begin{tabular}{|c|c|c|c|c|c|c|c|}
\hline & & $\begin{array}{l}\text { áticos } \\
\text { óficos } \\
=80)\end{array}$ & $\begin{array}{c}\text { As } \\
\text { con s } \\
\text { (n }\end{array}$ & $\begin{array}{l}\text { ticos } \\
\text { repeso } \\
677\end{array}$ & & $\begin{array}{l}\text { áticos } \\
\text { esos } \\
=72)\end{array}$ & $\mathbf{p}$ \\
\hline & & & & & & & ק 0000 \\
\hline Asma no controlada (GINA) & 30 & $(37,5)$ & 45 & $(67,2)$ & 46 & $(63,9)$ & 0,0008 \\
\hline Síntomas diurnos ( $\geq 2$ veces a la semana) & 45 & $(56,3)$ & 43 & $(64,2)$ & 56 & $(77,7)$ & 0,005 \\
\hline Síntomas nocturnos & 55 & $(68,8)$ & 49 & $(73,1)$ & 56 & $(77,7)$ & 0,21 \\
\hline Tos con ejercicio & 62 & $(77,5)$ & 58 & $(86,6)$ & 61 & $(84,7)$ & 0,22 \\
\hline Consultas en urgencia por crisis de asma & 26 & $(32,5)$ & 26 & $(38,8)$ & 24 & $(33,3)$ & 0,89 \\
\hline Hospitalizaciones por crisis de asma & 10 & $(12,5)$ & 9 & $(13,4)$ & 7 & $(9,7)$ & 0,78 \\
\hline Dosis elevadas de corticoide inhalatorio & 5 & $(6,3)$ & 3 & $(4,5)$ & 7 & $(9,7)$ & 0,41 \\
\hline Uso de corticoides más $\beta 2$ agonista de acción prolongada & 36 & $(45)$ & 31 & $(46,5)$ & 36 & $(50)$ & 0,59 \\
\hline Espirometría alterada & 22 & $(27,5)$ & 31 & $(46,3)$ & 32 & $(44,4)$ & 0,03 \\
\hline $\mathrm{VEF}_{1}<80 \%$ & 4 & $(5)$ & 2 & (3) & 2 & $(2,7)$ & 0,46 \\
\hline Relación $\mathrm{VEF}_{1} / \mathrm{CVF}<80 \%$ & 12 & $(15)$ & 23 & $(34,3)$ & 21 & $(29,2)$ & 0,04 \\
\hline $\mathrm{FEF}_{25-75}<\mathrm{LIN}$ & 23 & $(28,8)$ & 31 & $(46,3)$ & 32 & $(44,4)$ & 0,04 \\
\hline Cambio $\operatorname{VEF}_{1} \geq 12 \%$ post-salbutamol & 14 & $(17,5)$ & 17 & $(25,4)$ & 20 & $(27,7)$ & 0,13 \\
\hline Cambio $\geq 30 \% \mathrm{FEF}_{25-75}$ post-salbutamol & 23 & $(28,8)$ & 23 & $(34,3)$ & 20 & $(27,7)$ & 0,91 \\
\hline
\end{tabular}

$\mathrm{VEF}_{1}$ : volumen espiratorio forzado en el primer segundo, CVF: capacidad vital forzada, $\mathrm{FEF}_{25-75}$ : flujo espiratorio forzado al $25-75 \%$ de la capacidad vital forzada, LIN: límite inferior de normalidad.

con exceso de peso tienen un porcentaje significativamente mayor de asma no controlada, síntomas diurnos, espirometría alterada, relación $\mathrm{VEF}_{1} /$ CVF menor a $80 \%$ y de $\mathrm{FEF}_{25-75}$ bajo el límite inferior de normalidad que los asmáticos eutróficos.

La Tabla 3 muestra una tendencia estadísticamente significativa en el aumento de la sintomatología diurna, del asma no controlada y el deterioro de la función pulmonar mientras mayor fue la severidad de la patología nutricional por exceso. En la sintomatología diurna se aprecia claramente una tendencia lineal al aumento, sin embargo, en el asma no controlada según GINA, en la espirometría alterada, en la relación $\mathrm{VEF}_{1} /$ CVF menor a $80 \%$ y el $\mathrm{FEF}_{25-75}$ menor al límite inferior de normalidad, sólo se puede demostrar tendencia sin establecer categóricamente la linealidad.

\section{Comentarios}

Se encontró una frecuencia de sobrepeso y obesidad en niños con asma persistente que alcanzó casi dos tercios de la muestra. Esto llama la atención, pero no es nuevo. Un estudio previo con una gran cohorte reportó casi $50 \%$ de sobrepeso y obesidad en asmáticos de origen hispano, transformándose en la etnia con mayor prevalencia en estas patologías, incluso mayor que en asmáticos afroamericanos ${ }^{25}$. Creemos que la elevada proporción de asmáticos con sobrepeso y obesidad, responde a la mejoría de las condiciones socioeconómicas de las últimas décadas y la occidentalización de la dieta, lo que ya había sido reportado en la población general escolar y adolescente de nuestro país ${ }^{4}$. Otro factor que podría explicar el exceso de peso en el grupo de niños asmáticos es su mala condición o forma física. $\mathrm{La}$ percepción de disnea o dificultad respiratoria con el ejercicio, que no necesariamente corresponde a sintomatología asmática, los lleva a restringir la actividad deportiva, favoreciendo el sedentarismo y el exceso de peso.

Encontramos una mayor frecuencia de sobrepeso y obesidad en los varones. Respecto a este punto, la literatura tiene controversias, algunos estudios han notificado mayor frecuencia de estas condiciones en adolescentes asmáticas, mientras que otros reportan mayor riesgo de obesidad mórbida y síndrome metabólico en varones con asma $^{26-28}$.

Los asmáticos con sobrepeso y obesidad alcanzaron un porcentaje inusualmente elevado de adherencia al tratamiento controlador, cercano a dos tercios en cada grupo. A nuestro parecer, el menor grado de control y la percepción de mayor sintomatología registrada en estos pacientes, los incentivaría a mejorar cumplimiento terapéutico. 
En el caso de los asmáticos eutróficos la adherencia fue menor, llegando sólo al 50\%. Pensamos que posiblemente los asmáticos con estado nutricional normal desarrollan una enfermedad más fácil de controlar, muchas veces sin la necesidad del tratamiento inhalatorio corticoidal, lo que podría explicar la adherencia más baja que el grupo anterior.

Es importante mencionar que los factores modificables relacionados con mal control encontrados en este estudio son frecuentes en la mayoría de los niños asmáticos, pero sin duda dificultan el manejo de la enfermedad, especialmente en aquellos con sobrepeso u obesidad.

Se demostró que el control de síntomas en el grupo de asmáticos con exceso de peso es peor que en los asmáticos eutróficos, en especial al medir los síntomas diurnos como tos y sibilancias. Este último aspecto ya se había demostrado en adultos, sin embargo, aún se mantienen controversias en el asma infantil. Existen publicaciones que no han encontrado relación entre obesidad y mal control de asma en niños, sin embargo otras han comunicado aumento de los síntomas respiratorios y menor número de días libres de síntomas. Probablemente esto se deba a la dificultad que tienen los pacientes asmáticos obesos en interpretar o percibir con precisión su sintomatología, lo que tiene mayor impacto en la población adolescente ${ }^{29-31}$. No encontramos diferencias en consultas de urgencia u hospitalizaciones, pese a que publicaciones recientes han reportado mayor frecuencia de crisis de asma en urgencia o en unidad de paciente crítico para aquellos pacientes con sobrepeso $\mathrm{u}$ obesidad al compararlos con controles con estado nutricional normal $^{32,33}$.

Se ha descrito que el incremento del IMC en los niños con asma se correlaciona con la disminución de la relación $\mathrm{VEF}_{1} / \mathrm{CVF}^{34}$. Algunos estudios han demostrado que los asmáticos con sobrepeso $\mathrm{u}$ obesidad tendrían además declinación del $\mathrm{VEF}_{1}, \mathrm{FEF}_{25-75 \%}$ y CVF al ser comparados con asmáticos con estado nutricional normal. La hipótesis fisiopatológica de esta alteración estaría justificada por la disminución del volumen corriente y la capacidad residual funcional, lo que conduciría a una reducción del estiramiento del músculo liso con la consecuente obstrucción del lumen bronquial ${ }^{35,36}$. En nuestro estudio se logró demostrar que los porcentajes de limitación obstructiva en cualquiera de sus grados (espirometría alterada), de la relación $\mathrm{VEF}_{1} / \mathrm{CVF}$ menor a $80 \%$ y del $\mathrm{FEF}_{25-75 \%}$ bajo límites de normalidad fueron significativamente mayores en el grupo de asmáticos con exceso de peso que en los eutrófi- cos. En las proporciones de asma no controlada (que incluye $\mathrm{VEF}_{1}$ ), espirometría alterada, $\mathrm{VEF}_{1} /$ CVF menor al $80 \%$ y $\mathrm{FEF}_{25-75 \%}$ menor a los límites de normalidad, se encontró una tendencia significativa al aumento al pasar de una categoría nutricional a otra, sin embargo, el claro aumento en el grupo de asmáticos con sobrepeso, no se reflejó con la misma magnitud en los asmáticos obesos. Pensamos que la explicación para este hallazgo se relaciona con los resultados de algunos estudios recientes que han encontrado falta de correlación entre el incremento del IMC y la declinación de algunos parámetros de la función pulmonar en niños asmáticos. En dichos estudios se ha postulado que en los niños asmáticos, el incremento del IMC reflejaría mejor el tamaño corporal, que el porcentaje de masa grasa corporal. Por lo anterior, algunos niños asmáticos con mayor tamaño corporal y de su caja torácica, tendrían correlación positiva con el aumento de la función pulmonar, especialmente con el $\mathrm{VEF}_{1}$ y la CVF. Esta condición ha permitido recomendar la medición del índice cintura/cadera en la evaluación nutricional de los niños asmáticos, por considerarlo un parámetro que representaría mejor la asociación entre el aumento de la grasa corporal y la disminución de la función pulmo$\operatorname{nar}^{37,38}$. Se debe mencionar tres limitaciones de la investigación: la primera fue la falta de medición de hiperreactividad bronquial por medio de test de metacolina. Tradicionalmente se considera a la obesidad como un factor de riesgo independiente para hiperreactividad en asmáticos, sin embargo, la evidencia más reciente en adultos y niños con asma, ha demostrado que esta hipótesis podría no ser real y tendría cada vez menos importancia clínica $^{39,40}$. Otra limitación fue la falta de medición de volúmenes pulmonares, principalmente de la capacidad residual funcional, que en los asmáticos obesos disminuye a expensas del volumen de reserva espiratorio ${ }^{41,42}$. La tercera y a nuestro parecer más relevante, fue la ausencia de evaluación metabólica de los pacientes, ya que recientes investigaciones demuestran que los niños asmáticos obesos tendrían mayores alteraciones del metabolismo de glucosa, lípidos y riesgo de síndrome metabólico que aquellos sin obesidad ${ }^{43}$.

Los niños con asma tienen más sobrepeso y obesidad que aquellos sin asma, con mayores limitaciones en la capacidad para realizar actividad física y problemas emocionales secundarios. Además, en la transición de la adolescencia a la adultez, hay una notoria declinación de la actividad física aeróbica y anaeróbica, hecho que profundiza el sobrepeso y obesidad, con el consecuente empeoramiento de ambas patologías ${ }^{44-46}$. 
La pérdida de peso en pacientes con asma y obesidad tiene tres efectos fisiopatológicos teóricos: disminuye la inflamación sistémica y de la vía aérea, evita la contracción del músculo liso bronquial y mejorara la mecánica de la pared torácica. Estudios realizados en adultos con asma y obesidad han demostrado que los programas de reducción de peso basados en dietas hipocalóricas, así como algunas técnicas de cirugía bariátrica logran disminuir la percepción de disnea medida por escalas, la medicación de rescate $\mathrm{y}$ mejoran la función pulmonar ${ }^{47}$. Esto aún no ha sido investigado en niños o adolescentes, por lo que sería de mucha utilidad que las unidades de enfermedades respiratorias pediátricas desarrollen estudios de terapia, evaluando la respuesta clínica a programas de actividad física controlada o intervenciones nutricionales supervisadas.

Finalmente, concordamos que la evidencia mostrada en este estudio es preocupante, lo que obliga al pediatra especialista en enfermedades respiratorias a mantener una vigilancia nutricional periódica de los niños asmáticos, fomentar la actividad física y hábitos de vida saludable e incorporar a profesionales especializados en nutrición y entrenamiento en la atención de los pacientes.

\section{Bibliografía}

1.- OGDEN C, FLEGAL K, CARROLL M, JOHNSON C. Prevalence and trends in overweight among U.S. children and adolescents, 1999-2000. JAMA 2002; 288 : 1728-32.

2.- HEDLEY A, OGDEN C, JOHNSON C, CARROLL M, CURTIN L, FLEGAL K. Prevalence of overweight and obesity among US children, adolescents, and adults, 1999-2002. JAMA 2004; 291: 2847-50.

3.- OGDEN C, CARROLL M, FLEGAL K. High Body Mass Index for Age Among US Children and Adolescents, 2003-2006. JAMA 2008; 299: 2401-05.

4.- MUZZO S, BURROWS R, CORDERO J, RAMÍREZ I. Trends in Nutritional Status and Stature Among SchoolAge Children in Chile. Nutrition 2004; 20: 867-72.

5.- PEARCE N, AÎT-KHALED N, BEASLEY R, MALLOL J, KEIL U, MITCHELL E, et al. Worldwide trends in the prevalence of asthma symptoms: phase III of the International Study of Asthma and Allergies in Childhood (ISAAC). Thorax 2007; 62: 757-65.

6.- MALlOL J, AGUiRRE V, AGUILAR P, CALVO M, AMARALES L, ARELLANO P, et al. Changes in the prevalence of asthma in Chilean school age children between 1994 and 2002. International Study of Asthma and Allergies in Childhood (ISAAC)-Chile phases I and III. Rev. Méd. Chile 2007; 135: 580-6.
7.- CASTRO-RODRÍGUEZ J, HOLBERG C, MORGAN W, WRIGHT A, MARTÍNEZ F. Increased incidence of asthma-like symptoms in girls who become overweight or obese during the school years. Am J Respir Crit Care Med. 2001; 163: 1344-9.

8.- NOAL R, MENEZES A, MACEDO S, DUMITH S. Childhood body mass index and risk of asthma in adolescence: a systematic review. Obes Rev 2011; 12 (2): 93-104.

9.- BURGESS J, WALTERS E, BYRNES G, GILES G, JENKINS M, ABRAMSON M, et al. Childhood adiposity predicts adult-onset current asthma in females: a 25-yr prospective study. Eur Respir J 2007; 29: 668-75.

10.- MATRICARDI P, GRÛBER C, WAHN U, LAU S. The asthma-obesity link in childhood: open questions, complex evidence, a few answers only. Clinical and Experimental Allergy 2007; 37: 476-84.

11.- LITONJUA A, GOLD D. Asthma and obesity: Common early-life influences in the inception of disease. J Allergy Clin Immunol 2008; 121: 1075-84.

12.- KIM K, SHIN Y, LEE K, KIM E, SOHN M, KIM K. Relationship between adipokines and manifestations of childhood asthma. Pediatr Allergy Immunol 2008; 19 : 535-40.

13.- CASTRO-RODRÍGUEZ J. Relación entre obesidad y asma. Arch Bronconeumol 2007; 43: 171-5.

14.- STORY R. Asthma and obesity in children. Curr Opin Pediatr 2007; 19: 680-4.

15.- PETERS-GOLDEN M, SWERN A, BIRD S, HUSTAD C, GRANT E, EDELMAN J. Influence of body mass index on the response to asthma controller agents. Eur Respir J 2006; 27: 495-503.

16.- LAVOIE K, BACON S, LABRECQUE M, CARTIER A, DITTO B. Higher BMI is associated with worse asthma control and quality of life but not asthma severity. Respiratory Medicine 2006; 110: 648-57.

17.- SAINT-PIERRE P, BOURDIN A, CHANEZ P, DAURES J, GODARD P. Are overweight asthmatics more difficult to control? Allergy 2006; 61: 79-84.

18.- SHORE S. Obesity and asthma: implications for treatment. Curr Opin Pulm Med 2007; 13: 56-62.

19.- GINDE A, SANTILLAN A, CLARK S, CAMARGO C. Body mass index and acute asthma severity among children presenting to the emergency department. Pediatr Allergy Immunol 2010; 21: 480-8.

20.- VAN GENT R, VAN DER ENT C, ROVERS M, KIMPEN J, VAN ESSEN-ZANDVLIET L, DE MEER G. Excessive body weight is associated with additional loss of quality of life in children with asthma. J Allergy Clin Immunol 2007; 119: 591-6.

21.- PIZARRO T, RODRÍGUEZ L, BENAVIDES X. Norma Técnica de Evaluación Nutricional del Niño de 6 a 18 años. Año 2003. Rev Chil Nutr 2004; 31: 28-37.

22.- GLOBAL INITIATIVE FOR ASTHMA, GLOBAL STRATEGY FOR ASTHMA MANAGEMENT AND PREVENTION. Diagnosis and classification. Chapter 
2. Updated 2010. Available at: http://www.ginasthma. org [Consultado el 23 de diciembre de 2010].

23.- LINARES M, SÁNCHEZ I, CORRALES R, DÍAZ A, ESCOBAR A. Pruebas de función pulmonar en el niño. Rev Chil Pediatr 2000; 71: 228-42.

24.- GUTIÉRREZ M, BEROIZA T, BORZONE G, CAVIEDES I, CÉSPEDES J, GUTIÉRREZ M, et al. Espirometría: Manual de procedimientos. Sociedad Chilena de Enfermedades Respiratorias 2006. Rev Chil Enf Respir 2007; 23: 31-42.

25.- BENDER B, FUHLBRIGGE A, WALDERS N, ZHANG L. Overweight, Race, and Psychological Distress in Children in the Childhood Asthma Management Program. Pediatrics 2007; 120: 805-13.

26.- KATTAN M, KUMAR R, BLOOMBERG G, MITCHELL H, CALATRONI A, GERGEN P, et al. Asthma control, adiposity, and adipokines among inner-city adolescents. J Allergy Clin Immunol 2010; 125: 584-92.

27.- COTTRELL L, NEAL W, ICE C, PÉREZ M, PIEDIMONTE G. Metabolic Abnormalities in Children with Asthma. Am J Respir Crit Care Med 2011; 183: 441-8.

28.- DEL-RÍO-NAVARRO B, CASTRO-RODRÍGUEZ J, GARIBAY NIETO N, BERBER A, TOUSSAINT G, SIENRA-MONGE J, et al. Higher metabolic syndrome in obese asthmatic compared to obese nonasthmatic adolescent males J Asthma 2010; 47: 501-6.

29.- KWONG K, RHANDHAWA I, SAXENA J, MORPHEW T, JONES C. Ability to control persistent asthma in obese versus non-obese children enrolled in an asthma-specific disease management program (breathmobile). J Asthma 2006; 43: 661-6.

30.- HE Q, WONG T, DU L, JIANG Z, QIU H, GAO Y, et al. Respiratory health in overweight and obese Chinese children. Pediatr Pulmonol 2009; 44: 997-1002.

31.- KOPEL S, WALDERS-ABRAMSON N, MCQUAID E, SEIFER R, KOINIS-MITCHELL D, KLEIN R, et al. Asthma symptom perception and obesity in children. Biol Psychol 2010; 84: 135-41.

32.- CARROLL C, STOLTZ P, RAYKOV N, SMITH S, ZUCKER A. Childhood Overweight Increases Hospital Admission Rates for Asthma. Pediatrics 2007; 120: 734 40 .

33.- CARROLL C, UYGUNGIL B, ZUCKER A, SCHRAMM C. Identifying An At-Risk Population of Children With Recurrent Near-Fatal Asthma Exacerbations. J Asthma 2010; 47: 460-64.

34.- TANTISIRA K, LITONJUA A, WEISS S, FUHLBRIGGE A. Association of body mass with pulmonary function in the Childhood Asthma Management Program (CAMP). Thorax 2003; 58: 1036-41.

35.- LANG J, FENG H, LIMA J. Body mass index-percentile and diagnostic accuracy of childhood asthma. J Asthma 2009; 46: 291-9.

36.- SPATHOPOULOS D, PARASKAKIS E, TRYPSIANIS G, TSALKIDIS A, ARVANITIDOU V, EMPORIADOU $\mathrm{M}$, et al. The Effect of Obesity on Pulmonary Lung
Function of School Aged Children in Greece. Pediatr Pulmonol 2009; 44: 273-80

37.- PÉREZ-PADILLA R, ROJAS R, TORRES V, BORJAABURTO V, OLAIZ G. The Empece Working Group. Obesity among children residing in Mexico City and its impact on lung function: a comparison with MexicanAmericans. Arch Med Res 2006; 37: 165-71.

38.- CHEN Y, RENNIE D, CORMIER Y, DOSMAN J. Waist Circumference Associated With Pulmonary Function in Children. Pediatr Pulmonol 2009; 44: 216-21.

39.- DEESOMCHOK A, FISHER T, WEBB K, ORA J, LAM Y, LOUGHEED M, et al. Effects of obesity on perceptual and mechanical responses to bronchoconstriction in asthma. Am J Respir Crit Care Med 2010; 181: 125-33.

40.- MANSELL A, WALDERS N, WAMBOLDT M, CARTER R, STEELE D, DEVIN J, et al. Effect of body mass index on response to methacholine bronchial provocation in healthy and asthmatic adolescents. Pediatric Pulmonology 2006; 41: 434-40.

41.- BEUTHER D, WEISS S, SUTHERLAND E. Obesity and Asthma. Am J Respir Crit Care Med 2006; 174 112-9.

42.- JONES R, NZEKWU M. The effects of body mass index on lung volumen. Chest 2006; 130: 827-33.

43.- MCGINLEY B, PUNJABI N. Obesity, metabolic abnormalities, and asthma: establishing causal links. Am J Respir Crit Care Med 2011; 183: 424-5.

44.- BROCKMANN P, CAUSSADE S, HOLMGREN L, PRADO F, REYES B, VIVIANI P, et al. Actividad física y obesidad en niños con asma. Rev Chil Pediatr 2007; 78: 482-8.

45.- GLAZEBROOK C, MCPHERSON A, MACDONALD I, SWIFT J, RAMSAY C, NEWBOULD R, et al. Asthma as a Barrier to Children's Physical Activity: Implications for Body Mass Index and Mental Health. Pediatrics 2006; 18: 2443-9.

46.- WELSH L, ROBERTS R, KEMP J. Fitness and physical activity in children with asthma. Sports Med 2004; 34 : 861-70.

47.- JENSEN M, COLLINS C, GIBSON P, WOOD L. The Obesity Phenotype in Children with Asthma. Paediatric Respiratory Reviews 2011; 12: 152-9.

Correspondencia a:

Dr. Alberto Eduardo Vidal Grell

Dirección particular: Holanda No 87 Departamento 604/

Providencia / Santiago.

Fono fijo: 4152298 Fono celular: 9-6429701

E-mail: aevgmd@yahoo.es 